\title{
L'âge de la retraite en Chine
}

\section{Aiqing Zheng}

\section{(2) OpenEdition}

Journals

Édition électronique

URL : https://journals.openedition.org/rdctss/1252

DOI : $10.4000 /$ rdctss. 1252

ISSN : 2262-9815

\section{Éditeur}

Centre de droit comparé du travail et de la sécurité sociale

\section{Édition imprimée}

Date de publication : 1 avril 2020

Pagination : 166-169

ISSN : 2117-4350

\section{Référence électronique}

Aiqing Zheng, "L'âge de la retraite en Chine », Revue de droit comparé du travail et de la sécurité sociale [En ligne], 1 | 2020, mis en ligne le 01 novembre 2021, consulté le 11 novembre 2021. URL : http:// journals.openedition.org/rdctss/1252 ; DOI : https://doi.org/10.4000/rdctss.1252

\section{(c) (i) (9)}

Revue de droit comparé du travail et de la sécurité sociale est mise à disposition selon les termes de la Licence Creative Commons Attribution - Pas d'Utilisation Commerciale - Pas de Modification 4.0 International. 


\title{
AIQING ZHENG
}

\author{
FACUlté dE DROIT de RenMin, Université de ChINE
}

\section{L'ÂGE DE LA RETRAITE EN CHINE}

Un travailleur ayant dépassé l'âge de retraite mais qui est toujours activité, a-t-il encore le statut de salarié ? Un arrêt rendu en septembre de 2019 par la Cour supérieure de la province du Jiangsu, considéré comme l'un des 10 jugements les plus importants de 2019 sur le site internet du «droit du travail et de la sécurité sociale de Chine », a apporté une réponse positive à cette question. Estimant qu'il existait bien une relation de travail entre l'entreprise et la personne ayant dépassé l'âge de retraite mais encore en activité sur son poste, cet arrêt a ainsi donné gain de cause au travailleur qui revendiquait l'application du droit du travail à la rupture irrégulière de leur relation.

Cet arrêt fait écho aux nombreux litiges liés à l'âge de retraite, qui mettent en exergue les divergences de jurisprudence sur l'existence d'une relation de travail entre les deux parties. En pratique, pour le même type de procès, il existe différents jugements (I). Les carences des textes législatifs et du système de l'assurance retraite en Chine sont les principales raisons de ces divergences (II).

\section{I - LES LITIGES LIÉS À L'ÂGE DE DÉPART À LA RETRAITE}

En droit du travail chinois, l'arrivée à l'âge de la retraite constitue un des facteurs de rupture de la relation de travail. Dans ce cas, l'employeur procède aux formalités inhérentes et le travailleur concerné (le retraité) commence à percevoir sa pension mensuelle. Dès lors, la personne ayant atteint l'âge de la retraite n'a naturellement plus le statut de salarié. C'est un règlement du gouvernement central de 1978, adopté à l'époque de l'économie planifiée et n'ayant fait l'objet d'aucun amendement durant les trois dernières décennies, qui fixe l'âge de retraite légal à 60 ans pour les hommes, et à 55 ans - ou 50 ans - pour les femmes (selon qu'elles ont respectivement le statut de cadre ou d'ouvrière).

Ces dernières années, on constate un nombre croissant de procès directement liés à des travailleurs ayant dépassé l'âge de retraite, qui peuvent relever de l'une des trois catégories de litiges suivantes:

- le litige concerne l'entreprise et le travailleur qui est effectivement à la retraite et qui perçoit déjà sa pension ;

- le différend concerne l'entreprise et la personne qui, bien qu'ayant dépassé l'âge de départ à la retraite, reste à son poste ;

- le litige porte sur le fait qu'au moment du recrutement par l'entreprise, la personne a déjà dépassé l'âge de la retraite mais ne perçoit pas la pension normalement octroyée (cas du travailleur migrant)'.

S'agissant de la première catégorie de litiges entre des retraités et les entreprises qui les emploient, la pratique actuelle qui consiste à ce qu'un certain nombre de personnes "retraitées » travaillent encore dans les entreprises est une pratique gagnant-gagnant. En effet, d'une part ces retraités peuvent percevoir une rémunération en complément de leur pension mensuelle et,

1 S'ajoute une quatrième catégorie de litiges qui concerne l'inégalité de l'âge de retraite entre les femmes et les hommes, dont le présent article ne traite pas. 
d'autre part, les entreprises qui les emploient peuvent réduire le coût de la main-d'œuvre dans la mesure où, en dehors du salaire versé et convenu sur la base d'un accord mutuel, elles n'ont pas d'obligation de traitement au titre du droit du travail à l'instar des salariés " classiques». Toutefois, la survenue d'un accident sur le lieu du travail peut déclencher un différend : le retraité réclame tous les traitements dus, estimant que son accident doit être considéré comme un accident du travail, alors que l'entreprise en réfute la prise en charge. Face aux divergences des juges locaux qui, pendant plusieurs années, n'ont pas répondu directement à la question de la nature de l'accident, la Cour suprême a considéré, en 2010, que la relation juridique entre les deux parties était une relation de service (non une relation de travail) soumise au droit civil (au contrat civil)2. II en résulte que l'accident dont la victime est une personne " retraitée " n'a pas lieu d'être considéré comme un accident du travail. La victime de l'accident ne peut donc revendiquer que des réparations au nom de la responsabilité délictuelle civile.

Par ailleurs, ces dernières années, un autre type de procès se multiplie, engendré par des litiges entre l'entreprise et la personne qui a dépassé l'âge de la retraite mais qui ne perçoit pas la pension et demeure à son poste de travail. La situation se présente souvent de la façon suivante : un(e) salarié(e), soit un homme d'une soixantaine d'années ou une femme d'une cinquantaine d'années, parvient à l'âge de départ à la retraite, mais l'entreprise qui l'emploie n'a pas procédé aux formalités de retraite et de ce fait, il (elle) continue de travailler à son poste. Les litiges se déclenchent le plus souvent lors de la survenue d'un accident de travail. En effet, quelle est la nature de cet accident ? Est-ce un accident du travail, soumis au droit du travail, ou bien un accident dont la responsabilité doit être recherchée en droit civil ? Ensuite, les litiges portent davantage sur la nature de la relation entre les deux parties, dans la mesure où le travailleur réclame souvent, en cas de licenciement, les traitements dus en vertu du droit du travail. La question qui se pose est alors identique : s'agit-il d'une relation de travail soumise au droit du travail ou bien d'une relation de service relevant du droit civil?

Entre 2013 et 2016, les premières réactions des juges face à ces procès relatifs à un accident sur le lieu de travail, ont consisté à refuser, à la quasi-unanimité, de les qualifier d'accident du travail. Les juges estimaient en effet qu'à partir de la date préfigurant l'âge de la retraite, même si la personne travaille encore sur son poste, elle n'a plus le statut de salarié soumis au droit du travail, la relation juridique entre elle et son entreprise étant une relation de droit civil ${ }^{3}$. II en résulte l'application du principe de la responsabilité délictuelle selon lequel celui (ou celle) à qui incombe la responsabilité de l'accident est chargé(e) de dédommager les dégâts causés. Ainsi, il arrivait fréquemment que la personne accidentée soit responsable de son propre accident. Evidemment, cette situation était particulièrement perverse pour toutes les personnes de 50 à 60 ans encore en activité et ne percevant pas encore leur pension, victimes d'un accident sans pouvoir bénéficier des garanties liées à un accident du travail.

Cette préoccupation a été rapportée au ministère chargé du travail qui, en 2016, a proposé une autre interprétation du règlement sur l'assurance accident du travail, selon laquelle l'accident est qualifié d'accident du travail. Depuis, l'entreprise est responsable de l'accident du travail causé à cette catégorie de travailleurs et les frais médicaux sont remboursés. Cette interprétation ministérielle a constitué un progrès important vis-à-vis de ces travailleurs. Cependant, elle ne porte que sur l'accident du travail et ne répond pas à la question liée à l'existence ou non d'une relation de travail.

2 Art. 7 de la Troisième interprétation sur l'application des lois en matière du traitement des litiges au travail par la Cour suprême (12 juillet 2010) : le litige entre l'entreprise et la personne qui perçoit déjà sa pension - ou bénéficie déjà d'un traitement de retraite, doit être qualifié de litige associé à une relation de service (laowu guanxi).

3 Selon les statistiques établis par l'auteur concernant les jugements de 2015 en la matière. 
A cet égard, l'arrêt rendu en septembre 2019 par la Cour supérieure de la province du Jiangsu marque une étape majeure en confirmant l'existence d'une relation de travail entre l'entreprise et le travailleur ayant dépassé l'âge de la retraite, sans pour autant être parti à la retraite, et qui travaille encore dans l'entreprise. Cet arrêt a cassé les jugements de première et deuxième instance au motif d'une mauvaise interprétation de l'article 21 du règlement d'application de la loi sur le contrat de travail. La particularité de cet arrêt réside en outre dans le fait qu'il fait figure de jugement en procédure de révision ; or, très peu d'arrêts ayant force exécutoire peuvent faire l'objet de cette procédure.

Enfin, il existe une troisième catégorie de litiges, qui concerne la situation au cours de laquelle, lors du recrutement par l'entreprise, le travailleur - souvent un(e) travailleur migrant est déjà âgé de 50 ou 60 ans (selon qu'il est une femme ou un homme). Dans ce cas, quelle est la nature juridique de leur relation? Est-il légalement possible pour l'entreprise de recruter un(e) travailleur migrant de 50-60 ans dans la cadre d'une relation de travail établie? Courante dans le secteur du bâtiment, cette pratique permet de recruter de la main-d'œuvre à la campagne, souvent des séniors qui ne bénéficient pas d'une pension normale comme celle des retraités "citadins ». Ici encore, les litiges surviennent principalement en cas d'accident ou de renvoi du travailleur (licenciement).

Face à la question de l'accident survenu sur le lieu de travail, dans le secteur du bâtiment, le ministère du travail a progressivement mis en place, depuis 2014, une résolution obligeant l'entreprise - selon le projet de construction - à souscrire à une assurance accident du travail pour tous ses travailleurs, y compris les travailleurs migrants séniors. Quant à la qualification de leur relation, force est de constater une divergence des jugements en la matière ; une majorité refuse de la qualifier de relation de travail au motif du dépassement de l'âge de la retraite ${ }^{4}$. Ces travailleurs sont donc moins protégés sur le plan du droit du travail que les travailleurs citadins du même âge.

\section{II - LES CARENCES DES TEXTES ET DU SYSTÈME DE L'ASSURANCE RETRAITE}

À première vue, ces procès et divergences de jugements témoignent de l'absence de clarté de la législation du travail sur ces sujets. En effet, il existe un conflit - ou au moins une différence - entre l'article 44 de la loi sur le contrat de travail de 2007 et l'article 21 du règlement d'application de la loi sur le contrat de travail de 2008. Conformément à l'article 44, le fait de percevoir la pension de retraite constitue l'une des situations légales dans lesquelles le contrat de travail est rompu, alors que l'article 21 du règlement d'application de cette loi prévoit que l'arrivée de l'âge de la retraite est une circonstance qui permet de rompre le contrat de travail. De toute évidence, ces deux articles concernent des situations différentes puisque le moment de percevoir la pension est postérieur à l'arrivée de l'âge de la retraite. Le fait de recevoir une pension de retraite résulte de formalités effectuées par l'entreprise au profit du salarié en âge de prendre sa retraite. S'agissant du conflit - ou de la différence - entre ces deux dispositions, on peut considérer que le statut de la loi est théoriquement supérieur à celui du règlement. Cependant, il n'existe pas d'éclairages ou d'interprétations du législateur sur ce point. II résulte de ce flou législatif des divergences de jugements puisque certains ont fondé leur décision sur l'article 44, quand d'autres se sont basés sur l'article $21^{5}$. Quant au premier type susmentionné de litiges - entre l'entreprise et un retraité - et en dépit de l'interprétation de la Cour suprême de 2010, on observe encore des divergences de jugements en 2019.

4 Selon les statistiques établis par l'auteur concernant les arrêts rendus en la matière en 2019.

5 Selon les statistiques établis par l'auteur concernant les arrêts rendus en 2017, 2018 et 2019. 
Sur le fond, ces problématiques sont directement liées à deux considérations selon lesquelles l'établissement de l'âge de la retraite et l'assurance retraite en Chine sont déficients.

Le premier défaut concerne le départ forcé à la retraite dès lors qu'arrive l'âge de la retraite, sans considération des volontés du salarié ou de l'entreprise. L'âge de la retraite, fixé en 1978, est appliqué de manière automatique : l'entreprise met immédiatement le salarié à la retraite sans recueillir son avis. Dans un contexte aujourd'hui complètement différent de celui des années 1970, cette manière abrupte de « retraiter » les salariés devrait être remise en question.

Depuis 2014, face au vieillissement de la population, de nombreuses discussions académiques sont engagées pour instaurer plus de souplesse dans le système de retraite. Ainsi, Shanghai a mis en place une politique assouplie du système de retraite depuis 2013 : un accord entre l'entreprise et le salarié ayant dépassé l'âge de la retraite permet désormais au travailleur de rester en poste, sur la base d'une relation de travail spécifique maintenue et avec pour seule différence le fait que le salarié devra apporter sa contribution à l'assurance accident du travail. Sur le plan national cependant, malgré la certitude de l'allongement inéluctable, dans un futur proche, de l'âge de la retraite, le pays n'envisage pas d'apporter davantage de flexibilité à l'âge de départ à la retraite, ni même de généraliser la possibilité d'un départ à la retraite basé sur les volontés du salarié, notamment parce que les volontés varient beaucoup d'une catégorie socioprofessionnelle à une autre, ou d'un secteur à l'autre ${ }^{6}$.

La deuxième considération porte sur l'existence - ou non - d'une limite d'âge maximale des personnes régies par le droit du travail, au-delà de laquelle la personne n'a plus le statut de salarié, ou ne peut plus bénéficier de la pension de retraite.

Il est clair que les personnes retraitées et donc déjà bénéficiaires de la pension ne sont plus protégées par le droit du travail lorsqu'elles continuent de travailler pour l'entreprise. Cependant, en Chine, les travailleurs migrants (principalement d'origine rurale) ont le plus souvent dépassé l'âge de 50 ou 60 ans et, de ce fait, appartiennent à la catégorie des personnes retraitées. Mais l'assurance retraite n'est pas obligatoire pour eux, contrairement aux salariés citadins ${ }^{7}$. Leur pension, qui consiste plutôt en une sorte de subvention gouvernementale, représente une somme symbolique qui ne leur permet pas de vivre et les oblige à travailler (quand leur état de santé le permet). Or, ces travailleurs migrants séniors ne devraient pas être exclus de la protection du droit du travail tant que leur assurance retraite déficiente ne s'est pas améliorée.

Il reste à espérer que le gouvernement central adoptera, dans un futur proche, une politique souple vis-à-vis du départ à la retraite, afin de mieux répondre aux besoins des entreprises et aux souhaits des différents groupes de travailleurs sur le sujet de la retraite. Du point de vue de la sécurité juridique, il est nécessaire que le législateur et la justice parviennent à des interprétations analogues pour que disparaissent ces divergences jurisprudentielles.

6 Depuis 2014, il existe de nombreuses discussions pour assouplir l'âge de départ à la retraite, et pour allonger l'âge de retraite, mais il semblerait que l'on n'ait pas encore trouvé de consensus.

7 Malgré un progrès très significatif de la couverture sociale en Chine depuis 2012, qui permet à la population âgée rurale de percevoir mensuellement une somme d'argent, leur assurance retraite, volontaire, fondée sur les subventions gouvermentales, reste nettement différente de celle des salariés en ville. 